Proc. 13th International School on Theoretical Physics: Symmetry and Structural Properties of Condensed Matter

\title{
Current Induced Dynamics of One-Dimensional Skyrmions
}

\author{
M. SedlmayR*, N. Sedlmayr and V.K. DugaeV \\ Department of Physics and Medical Engineering, Rzeszów University of Technology, \\ al. Powstańców Warszawy 6, 35-959 Rzeszów, Poland
}

\begin{abstract}
Magnetic textures in ferromagnets are not confined only to the appearance of domain walls, but can also include more complicated structures such as one-dimensional skyrmions. Such different magnetization textures can give rise not only to different magnetoresistance effects, but also to different dynamics of the various magnetic textures caused by applied currents and fields. In this article we investigate the dynamics of different magnetic textures caused by applied currents in the wires.
\end{abstract}

DOI: 10.12693/APhysPolA.135.1268

PACS/topics: domain wall, magnetisation dynamics, skyrmions

\section{Introduction}

Skyrmions are well known topological objects which can form, under certain conditions, in 2D magnetic condensed matter systems [1-7]. The topology is defined by an appropriate winding number of the magnetic order. In $1 \mathrm{D}$ materials it is also possible to find a variety of different magnetic twists, separating regions of collinear magnetization. These can also be protected "topologically" between bulk orientations. Indeed, one can even define a winding number to categorise them. One potential interest in skyrmions is the view of using them as bits in a spintronic device. Skyrmions in thin planar systems could be thought of as quasi 1D. Here we consider the fully 1D limit of such possible topological objects. This is naturally of relevance in the continuing drive to make devices ever smaller and more efficient. For potential spintronics devices [8, 9], and also of fundamental physical interest, is how these structures can be manipulated, and what kind of transport signal they have. Such skyrmion-like structures would also arise when domain walls of the appropriate configuration are joined.

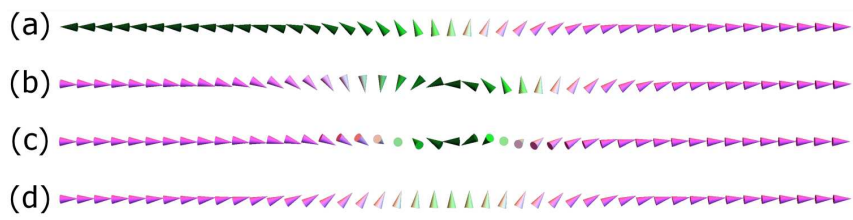

Fig. 1. Schematics of the magnetic textures. Each cone represents the magnetization at its local position, but not all lattice sites are shown. The colour relates to the direction of the magnetization in the $x$ $z$ plane. Part (a) is a domain wall (6), with $\nu=\frac{1}{2}$, (b) is a skyrmion (5), with $\nu=1$, (c) is an out-of-plane skyrmion (8), with $\nu=1$, (d) is a domain wall, see (7), with $\nu=0$.

*corresponding author; e-mail: m.sedlmayr@prz.edu.pl
Manipulation of domain walls can be caused by applying a current and their dynamics is the subject of many studies. Some examples can be found in Refs. [10-14]. Here we focus on the current induced motion of both stable and unstable configurations of one dimensional magnetic skyrmions. Schematics of the magnetisation textures under consideration are in Fig. 1.

\section{Model}

We use a standard $s$-d approximation in which the time scales of the fast conduction electrons and slow bulk magnetisation are assumed to be separable. The dynamics of the magnetisation can be solved using the Landau-Lifshitz-Gilbert equations with an additional phenomenological relaxation term [15] and coupling to current $\boldsymbol{j}_{e}$ which includes non-adiabatic torque from spin relaxation [16]:

$$
\begin{gathered}
\partial_{t} \boldsymbol{M}=-\frac{1}{1+\alpha^{2}}\left[\gamma \boldsymbol{M} \times \mathcal{H}+\frac{\alpha \gamma}{M} \boldsymbol{M} \times(\boldsymbol{M} \times \mathcal{H})\right. \\
+\frac{b_{j}(1+\alpha \xi)}{M^{2}} \boldsymbol{M} \times\left(\boldsymbol{M} \times\left(\boldsymbol{j}_{e} \cdot \nabla\right) \boldsymbol{M}\right) \\
\left.+\frac{b_{j}(\xi-\alpha)}{M} \boldsymbol{M} \times\left(\boldsymbol{j}_{e} \cdot \nabla\right) \boldsymbol{M}\right] .
\end{gathered}
$$

$\alpha$ is the phenomenological damping term and $\gamma=g \mu_{\mathrm{B}} / \hbar$ is the gyromagnetic ratio, where $g=2$ is the Landé factor, and $\mu_{\mathrm{B}}$ is the Bohr magneton. $\xi=\tau_{e x} / \tau_{s f}$ is the ratio between the exchange and spin-flip time scales, and $b_{j}=P \mu_{\mathrm{B}} /\left[e M\left(1+\xi^{2}\right)\right]$, where $P$ is the polarisation of the conduction electrons, $e-$ the electron charge, and $M$ - the magnitude of the magnetisation. Furthermore $\mathcal{H}$ is the effective field

$$
\mathcal{H}=J \boldsymbol{S}+\alpha_{\mathrm{ex}} \frac{\partial^{2} \boldsymbol{M}}{\partial x^{2}}+\boldsymbol{K} \cdot \boldsymbol{M}+\boldsymbol{B} .
$$

$f_{0}(M)$ is the homogeneous exchange term, $\alpha_{\mathrm{ex}}$ is the exchange coupling, and $\boldsymbol{K}$ is the magnetic anisotropy which we can write in a convenient notation as $\boldsymbol{K}=$ $\left(K_{x} \hat{x}, K_{y} \hat{y}, K_{z} \hat{z}\right)$ so that $\boldsymbol{K} \cdot \boldsymbol{M}$ remains a vector quantity. $J$ is the coupling between the bulk magnetization $\boldsymbol{M}$ and the spin of the conduction electrons $\boldsymbol{S}$. The profile of $\boldsymbol{S}$ in the adiabatic limit will be parallel to $\boldsymbol{M}$ though 
both interaction effects important in one dimension can potentially change this $[17,18]$ as well as current induced interactions between different magnetic textures present in the wire [19]. An appropriate applied magnetic field $\boldsymbol{B}$ could be used to help stabilise some of the skyrmions from distortion or breaking apart, but here we will focus on the case $\boldsymbol{B}=\mathbf{0}$.

We consider the 1D magnetic wire with a certain profile of magnetization $\mathbf{M}(\mathbf{x})$ which typically changes direction in the $x-z$ plane, with $y$ being a hard axis. The hard axis ensures some protection for the different magnetic profiles. Assuming that rotation of the magnetisation into the $y$ direction is completely forbidden, the magnetic structures possess topological protection exactly as for two-dimensional skyrmions.

The magnetisation can be written as $\boldsymbol{M}=M \hat{n}$ with $\hat{n}=(\sin [\phi(x)], \cos [\phi(x)] \sin [\theta(x)], \cos [\phi(x)] \cos [\theta(x)])$.

The winding number for the magnetic texture is

$$
\nu=\frac{1}{2 \pi} \int_{-\infty}^{\infty} \frac{\mathrm{d} n_{x}}{\mathrm{~d} x} \mathrm{~d} x .
$$

If $\lim _{x \rightarrow \infty} n_{x}(x)=\lim _{x \rightarrow-\infty} n_{x}(x)$ then this must be an integer. Note that this condition is not satisfied for a domain wall. In one dimension one can find various configurations for a magnetic texture between bulk collinear magnetisation. Here we will focus on four different configurations, or magnetisation textures, as initial conditions of the dynamics, two stable and two potentially unstable. See Fig. 1 for schematics of the configurations. We use two different skyrmions. Firstly an energetically favourable skyrmion $(\nu=1)$ confined to the $x-z$ plane with $\theta_{S}(x)=0$ and

$$
\phi_{S}(x)=\frac{\pi}{2}-2 \cos ^{-1}\left(\tanh \left(\frac{x}{\lambda}\right)\right) .
$$

As a comparison we consider a domain wall between two regions of opposite magnetisation direction $(\nu=1 / 2)$ with $\theta_{D W}(x)=0$ and

$$
\phi_{D W}(x)=\frac{\pi}{2}-\cos ^{-1}\left(\tanh \left(\frac{x}{\lambda}\right)\right) .
$$

Additionally we have an unstable and unprotected impurity-like magnetic texture $(\nu=0)$ with $\theta_{I}(x)=0$ and

$$
\phi_{I}(x)=\frac{\pi}{2}-\cos ^{-1}\left(\tanh ^{2}\left(\frac{x}{\lambda}\right)\right) .
$$

Finally, we consider a skyrmion $(\nu=1)$ which is rotated through all directions where

$$
\begin{aligned}
& \theta_{S O P}(x)=2 \cos ^{-1}\left(\tanh \left(\frac{x}{\lambda}\right)\right), \\
& \text { and } \phi_{S O P}(x)=\frac{\pi}{2}-2 \cos ^{-1}\left(\tanh \left(\frac{x}{\lambda}\right)\right) .
\end{aligned}
$$

As this configuration is already twisted into the hard axis, it is not clear that its structure is protected by any topology.

In the following calculations we will use the following values, relevant for thin metallic nanowires. The Fermi wavelength is $\lambda_{\mathrm{F}}=0.367 \mathrm{~nm}$, the DW length is $\lambda=80 \lambda_{\mathrm{F}}$, and $J M=0.2 \epsilon_{\mathrm{F}}$ with $M=6 \times 10^{27} \mu_{\mathrm{B}}$, where $\epsilon_{\mathrm{F}}=\hbar^{2} k_{F}^{2} / 2 m$ is the Fermi energy. $m$ is the electron mass and $k_{\mathrm{F}}=2 \pi / \lambda_{\mathrm{F}}$. For the spin channels we define $\epsilon_{\mathrm{F}} \pm J M=\hbar^{2} k_{\mathrm{F} \uparrow, \downarrow}^{2} / 2 m$ and the polarisation is $P=\left(k_{\mathrm{F} \uparrow}-k_{\mathrm{F} \downarrow}\right) /\left(k_{\mathrm{F} \uparrow}+k_{\mathrm{F} \downarrow}\right)$. When relevant the applied current is $j_{e}=-2 \times 10^{8} \hat{x} \mathrm{~A}$. Damping is taken to be $\alpha=0.05$ and we use $\xi=0.011$. The exchange coupling is $\alpha_{\text {ex }}=J / M a_{\text {lat }}$ where $a_{\text {lat }}=0.1 \mathrm{~nm}$ is the lattice spacing. Finally $K_{x}=K_{z}=0$ and $K_{y}=-\alpha_{\mathrm{ex}}(\pi / \lambda)^{2}$ is a hard magnetisation axis.

We will first check the stability of the configurations given by Eqs. (5)-(7) in the absence of an applied current. Both the domain wall and the skyrmion are stable. As can be seen in Fig. 2 the out-of-plane skyrmion and the impurity both relax, although it is not clear in the available time span what the long time limit for the configuration of the out-of-plane skyrmion is.

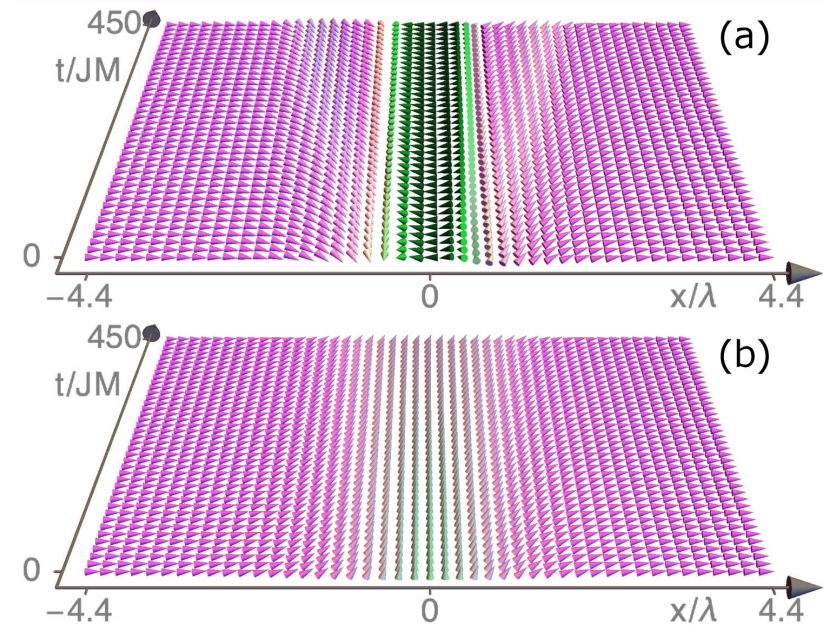

Fig. 2. Relaxation in the absence of an applied current, of (a) an out-of-plane skyrmion and (b) an impurity, see Eqs. (8) and (7), respectively. Both structures can be seen to be not fully stable. See Fig. 1 for an explanation of the figures.

The current induced motion of domain walls is now very well understood. The same mechanisms that set a domain wall in motion also apply to all of the magnetisation textures studied here. The principle mechanism for current induced motion studied here is the non-adiabatic torque from relaxation of the current electrons [16]. We start at time $t=0$ with the four configurations in Eqs. (5)-(7) and apply a constant current $j_{e}=-2 \times 10^{8} \hat{x} \mathrm{~A}$.

The domain wall moves as a coherent entity, without modifying its shape, as can be seen in Fig. 3a. The skyrmion moves with a similar velocity, but the profile is distorted by the motion, as shown in Fig. 3b. Although the skyrmion remains stable under these deformations, we cannot see what the long time behaviour is in the available time. Interestingly the current induced motion seems to prevent the decay of the magnetic impurity texture, as shown in Fig. 3d, making it much longer lived. 

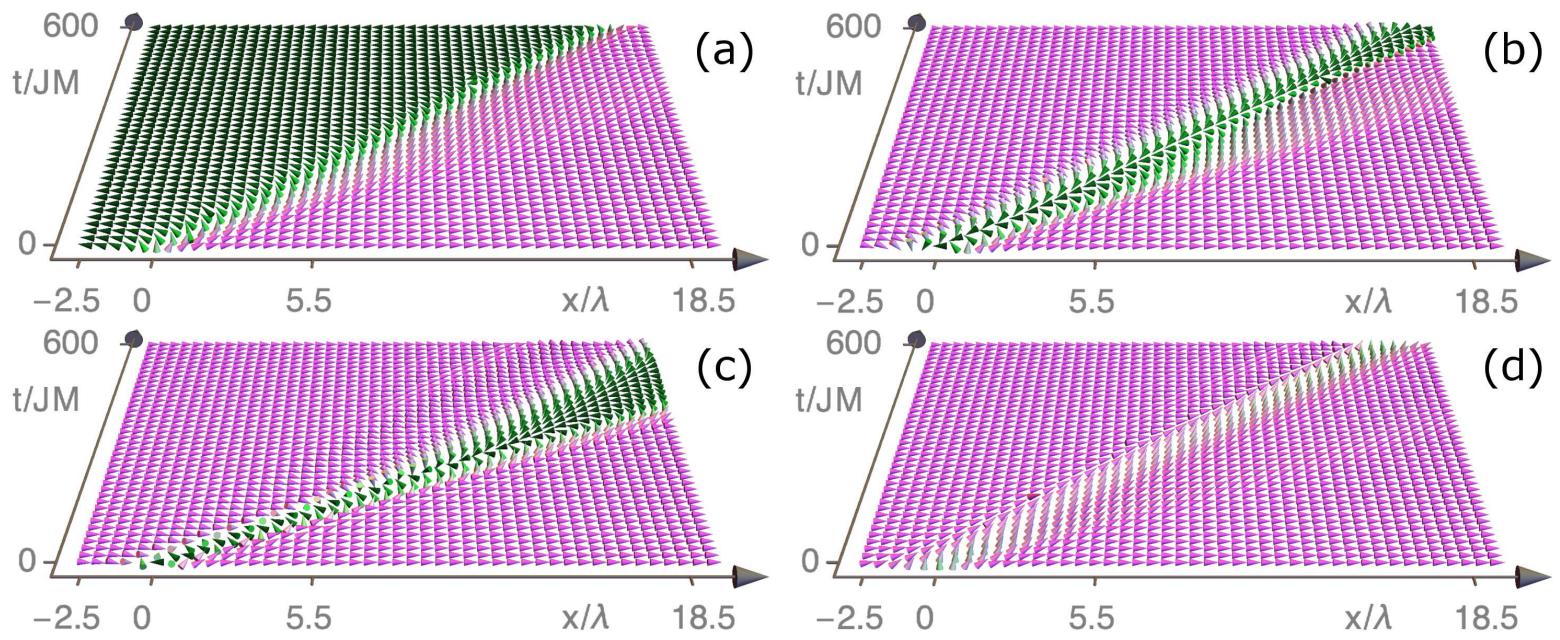

Fig. 3. Current induced dynamics of: (a) a skyrmion, (b) a domain wall, (c) an out-of-plane skyrmion, and (d) an impurity. One can see that when motion is induced it appears to delay the relaxation of the two unstable configurations (c) and (d). See Fig. 1 for an explanation of the figures.

The unstable out-of-plane skyrmion has a larger velocity than the other magnetisation textures, as shown in Fig. 3c. However it is more severely deformed by the motion. Even though it survives in the available time scale, it becomes spatially spread out, leaving a long "tail" behind it.

\section{Conclusions}

We have demonstrated that a $1 \mathrm{D}$ equivalent of a skyrmion in a narrow ferromagnetic wire can be stable and have studied its dynamics for constant applied currents. Contrary to various unstable configuration also tested we have shown that a skyrmion can undergo coherent motion without severely distorting its shape. In the future we plan to study in detail the motion of various potential skyrmion configurations, and additionally include the effects of pulsed currents, applied magnetic fields, and pinning centres. We also note that even unstable configurations like the impurity can be stabilised in the short term by the application of a current, which prevents them from relaxing.

\section{Acknowledgments}

This work was supported by the National Science Center in Poland as research Project No. UMO2017/27/B/ST3/02881 and by Rzeszów University of Technology (No. DS.FC.18.001/2018).

\section{References}

[1] C.A. Akosa, O.A. Tretiakov, G. Tatara, A. Manchon, Phys. Rev. Lett. 121, 97204 (2017).

[2] A. Fert, N. Reyren, V. Cros, Nature Rev. Mater. 2, 17031 (2017)
[3] X.Z. Yu, W. Koshibae, Y. Tokunaga, K. Shibata, Y. Taguchi, N. Nagaosa, Y. Tokura, Nature 564, 95 (2018).

[4] E.Y. Tsymbal, C. Panagopoulos, Nature Mater. 17, 1054 (2018)

[5] S. Hayami, Y. Motome, Phys. Rev. Lett. 121, 137202 (2018).

[6] L. Wang, Q. Feng, Y. Kim, R. Kim, K.H. Lee, S.D. Pollard, Y.J. Shin, H. Zhou, W. Peng, D. Lee, W. Meng, H. Yang, J.H. Han, M. Kim, Q. Lu, T.W. Noh, Nature Mater. 17, 1087 (2018).

[7] I. Lemesh, G.S.D. Beach, Phys. Rev. B 98, 104402 (2018).

[8] C.H. Marrows, Adv. Phys. 54, 585 (2005).

[9] S.S.P. Parkin, M. Hayashi, L. Thomas, Science 320, 190 (2008).

[10] A. Thiaville, J.M. Garcia, J. Miltat, J. Magn. Magn. Mater. 242, 1061 (2002)

[11] Y. Tserkovnyak, H.J. Skadsem, A. Brataas, G.E. Bauer, Phys. Rev. B 74, 144405 (2006).

[12] G. Bertotti, C. Serpico, I.D. Mayergoyz, R. Bonin, M. D'Aquino, J. Magn. Magn. Mater. 316, 285 (2007).

[13] L. Thomas, R. Moriya, C. Rettner, S.S. Parkin, Science 330, 1810 (2010).

[14] L. Heyne, J. Rhensius, A. Bisig, S. Krzyk, P. Punke, M. Kläui, L.J. Heyderman, L.L. Guyader, F. Nolting, Appl. Phys. Lett. 96, 032504 (2010).

[15] T. Gilbert, IEEE Trans. Magn. 40, 3443 (2004).

[16] S. Zhang, Z. Li, Phys. Rev. Lett. 93, 127204 (2004).

[17] M.A.N. Araújo, V.K. Dugaev, V.R. Vieira, J. Berakdar, J. Barnaś, Phys. Rev. B 74, 1 (2006).

[18] N. Sedlmayr, S. Eggert, J. Sirker, Phys. Rev. B $\mathbf{8 4}$ 24424 (2011).

[19] N. Sedlmayr, V.K. Dugaev, J. Berakdar, Phys. Rev. B 79, 174422 (2009); Phys. Rev. B 83, 174447 (2011); N. Sedlmayr, V.K. Dugaev, M. Inglot, J. Berakdar, Phys. Status Solidi Rapid Res. Lett. 5, 450 (2011). 